\title{
New Test Statistics for One and Two Mean Vectors with Two-step Monotone Missing Data
}

\author{
Mizuki Onozawa ${ }^{1}$, Ayaka Yagi $^{2}$, Takashi $\mathrm{Seo}^{2}$ \\ ${ }^{1}$ Showa Elementary School, Tokyo, Japan \\ ${ }^{2}$ Department of Applied Mathematics, Tokyo University of Science, Tokyo, Japan \\ Correspondence: Mizuki Onozawa, Showa Elementary School, Bunkyo Ward, Tokyo, Japan. \\ E-mail: onozawamizukil@gmail.com
}

Received: August 5, 2020 Accepted: September 8, 2020 Online Published: October 14, 2020

doi:10.5539/ijsp.v9n6p56 URL: https://doi.org/10.5539/ijsp.v9n6p56

\begin{abstract}
We consider the tests for a single mean vector and two mean vectors with two-step monotone missing data. In this paper, we propose new test statistics for one sample and two sample designs based on the simplified $T^{2}$-type test statistic. Further, we present the approximation to the upper percentiles of these statistics and propose the transformed test statistics. Finally, we investigate the accuracy and asymptotic behavior of the approximation for $\chi^{2}$ distribution by a Monte Carlo simulation.
\end{abstract}

Keywords: asymptotic expansion, Bartlett correction, $\chi^{2}$ approximation, Hotelling's $T^{2}$-type test statistic, monotone missing data, transformed test statistic

\section{Introduction}

The test for the equality of mean vectors has been discussed using the Hotelling's $T^{2}$ test statistic and likelihood ratio test statistic (see, e.g., Morrison (2005)). We often encounter the problem of missing data in many practical situations. In one sample problem, Jinadasa and Tracy (1992) obtained a closed form expression for the maximum likelihood estimators (MLEs) for the mean vector and the covariance matrix in the case of $k$-step monotone missing data. Krishnamoorthy and Pannala (1998) considered the likelihood ratio test statistic with $k$-step monotone missing data. Chang and Richards (2009) considered the $T^{2}$ test statistic for the mean vector with two-step monotone missing data. Further, Seko et al. (2012) discussed the $T^{2}$ test statistic and likelihood ratio test statistic using linear interpolation. Meanwhile, it is difficult to obtain an exact covariance matrix for the MLE of the mean vector since the $T^{2}$ test statistic is complicated with missing data. Therefore, Krishnamoorthy and Pannala (1999) provided a simplified $T^{2}$ test statistic and approximated the upper percentiles using the $F$ distribution. They adjusted the freedom of $F$ distribution using the expected value and the variance of the test statistic. They discussed $k$-step monotone missing data although they only provided MLEs with up to three-step monotone missing data. Yagi and Seo (2017) provided the approximate upper percentiles of the simplified $T^{2}$ test statistic using linear interpolation with the notation used by Jinadasa and Tracy (1992). Yagi et al. (2019) provided an asymptotic expansion for the null distribution of the simplified $T^{2}$ test statistic and improved the $\chi^{2}$ approximation for the statistic in the case of two and $k$-step monotone missing data. In a two sample problem, Yu et al. (2006) reported a simplified $T^{2}$-type test statistic with $k$-step monotone missing data using Krishnamoorthy and Pannala (1999)'s idea. Linear interpolation approximation for the null distribution of the Hotelling's $T^{2}$-type statistic and likelihood ratio test statistic with two-step monotone missing data were reported by Seko et al. (2011). Moreover, Yagi and Seo (2017) also considered a two sample problem, with the pivotal quantities similar to the Hotelling's $T^{2}$-type statistic in Yu et al. (2006). Yagi et al. (2018) extended the result of the one sample problem in their recent work. In this paper, we propose test statistics for one and two sample problems with two-step monotone missing data under consideration by Krishnamoorthy and Pannala (1998) and $\mathrm{Yu}$ et al. (2006). In a one sample problem, the part of the likelihood ratio in Krishnamoorthy and Pannala (1998) is used as the test statistics than we have. The two sample problem is considered in the same manner as that of the one sample problem. Further using the asymptotic expansion of the distribution, we present the transformed test statistics based on the Bartlett adjustment. The detailed explanation of the Bartlett adjustment is found in e.g., Anderson (2003). The improved transformations for the general test statistic are discussed by Fujikoshi (2000). The organization of this paper is as follows. In Section 2, we first present the assumption and notations. In Section 3, we derive an asymptotic expansion of the null distribution of the new test statistics. In Section 4, we consider transformed test statistics for the proposed test statistics and approximated upper percentiles of the distribution. In Section 5, we describe the Monte Carlo simulation that was implemented to investigate the accuracy for the null distributions of these statistics. Finally, in Section 
6, we conclude this study.

\section{Assumption and Notations}

Let us consider the $\ell$-th data set $\boldsymbol{X}^{(\ell)}$ that has the same monotone pattern as that of the two-step monotone missing data $(*$ : missing part $\ell=1,2)$ :

$$
\boldsymbol{X}^{(\ell)}=\left(\begin{array}{cc}
\boldsymbol{X}_{11}^{(\ell)} & \boldsymbol{X}_{12}^{(\ell)} \\
\boldsymbol{X}_{21}^{(\ell)} & *
\end{array}\right)=\left(\begin{array}{c}
\boldsymbol{X}_{1(12)}^{(\ell)} \\
\boldsymbol{X}_{21}^{(\ell)}
\end{array}\right),
$$

where $\boldsymbol{X}_{11}^{(\ell)}$ is a $n_{1}^{(\ell)} \times p_{1}$ block matrix, $\boldsymbol{X}_{12}^{(\ell)}$ is a $n_{1}^{(\ell)} \times p_{2}$ block matrix, $\boldsymbol{X}_{21}^{(\ell)}$ is a $n_{2}^{(\ell)} \times p_{1}$ block matrix, and $\boldsymbol{X}^{(\ell)}$ is independent and distributed as a multivariate normal distribution with a common covariance matrix. Next, we assume the distribution of the observation vectors in the following manner:

$$
\operatorname{vec}\left(\boldsymbol{X}_{1(12)}^{(\ell)^{\prime}}\right) \sim N_{n_{1}^{(\ell)} p}\left(\operatorname{vec}\left(\boldsymbol{\mu}^{(\ell)} \mathbf{1}_{n_{1}^{\prime}}^{\prime(\ell)}\right), \boldsymbol{I}_{n_{1}^{(\ell)}} \otimes \boldsymbol{\Sigma}\right), \quad \operatorname{vec}\left(\boldsymbol{X}_{21}^{(\ell)^{\prime}}\right) \sim N_{n_{2}^{(\ell)} p_{1}}\left(\operatorname{vec}\left(\boldsymbol{\mu}_{1}^{(\ell)} \mathbf{1}_{n_{2}^{\prime(\ell)}}^{\prime}\right), \boldsymbol{I}_{n_{2}^{(\ell)}} \otimes \boldsymbol{\Sigma}_{11}\right),
$$

respectively, where

$$
\boldsymbol{\mu}^{(\ell)}=\left(\begin{array}{c}
\boldsymbol{\mu}_{1}^{(\ell)} \\
\boldsymbol{\mu}_{2}^{(\ell)}
\end{array}\right), \boldsymbol{\Sigma}=\left(\begin{array}{cc}
\boldsymbol{\Sigma}_{11} & \boldsymbol{\Sigma}_{12} \\
\boldsymbol{\Sigma}_{12}^{\prime} & \boldsymbol{\Sigma}_{22}
\end{array}\right)
$$

and $\boldsymbol{\mu}^{(\ell)}$ and $\boldsymbol{\Sigma}$ are partitioned according to the blocks of the data set. Therefore, $\boldsymbol{\mu}_{j}^{(\ell)}(j=1,2)$ is a $p_{j}$-dimensional vector and $\boldsymbol{\Sigma}_{j m}(j, m=1,2)$ is a $p_{j} \times p_{m}$ matrix. Further, let $\boldsymbol{\mu}^{(\ell)}$ be the $p$ dimensional mean vector of $\boldsymbol{X}^{(\ell)}$, where $p=p_{1}+p_{2}$. Let $\overline{\boldsymbol{x}}_{(12) 1}^{(\ell)}$ be the sample mean vector, $\boldsymbol{S}_{(12) 1}^{(\ell)}$ be the unbiased sample covariance matrix of $\boldsymbol{X}_{(12) 1}^{(\ell)}$. Let $\overline{\boldsymbol{x}}_{1(12)}^{(\ell)}\left(=\left(\overline{\boldsymbol{x}}_{11}^{(\ell)^{\prime}}, \overline{\boldsymbol{x}}_{12}^{(\ell)^{\prime}}\right)^{\prime}\right)$ be the sample mean vector, and $\boldsymbol{S}_{1(12)}^{(\ell)}$ be the unbiased sample covariance matrix of $\boldsymbol{X}_{1(12)}^{(\ell)}$, where

$$
\left(\begin{array}{l}
\boldsymbol{X}_{11}^{(\ell)} \\
\boldsymbol{X}_{21}^{(\ell)}
\end{array}\right)=\boldsymbol{X}_{(12) 1}^{(\ell)}, \quad \boldsymbol{S}_{1(12)}^{(\ell)}=\left(\begin{array}{ll}
S_{1(12), 11}^{(\ell)} & S_{1(12), 12}^{(\ell)} \\
S_{1(12), 12}^{(\ell)^{\prime}} & S_{1(12), 22}^{(\ell)}
\end{array}\right) .
$$

\section{Asymptotic Expansion for the Distribution of the Test Statistic}

\subsection{One Sample Problem}

We first consider the one sample problem. Further, in this case, we define the notation of the dataset by omitting " $(\ell)$ " from superscript of the notation defined in the previous section, for example, $\boldsymbol{X}^{(\ell)}=\boldsymbol{X}$. Then, the simplified $T^{2}$-type statistic for the hypothesis is the following:

$$
H_{0}: \boldsymbol{\mu}=\boldsymbol{\mu}_{0} \text { vs. } H_{1}: \boldsymbol{\mu} \neq \boldsymbol{\mu}_{0}
$$

(without loss of generality, we can assume in (1) that $\boldsymbol{\mu}_{0}=\mathbf{0}$ ) is given by

$$
Q=Q_{1}+Q_{2}
$$

where

$$
\begin{aligned}
& Q_{i}=N_{i} \widehat{\boldsymbol{\eta}}_{i} \widehat{\Delta}_{i i}^{-1} \widehat{\boldsymbol{\eta}}_{i}, \quad N_{i}=\sum_{j=1}^{i} n_{j}, \quad i=1,2, \\
& \widehat{\boldsymbol{\eta}}_{1}=\overline{\boldsymbol{x}}_{(12) 1}, \quad \widehat{\boldsymbol{\eta}}_{2}=\overline{\boldsymbol{x}}_{12}-\boldsymbol{S}_{1(12), 21} \boldsymbol{S}_{1(12), 11}^{-1} \overline{\boldsymbol{x}}_{11}, \\
& \widehat{\Delta}_{11}=\frac{N_{2}-1}{N_{2}} \boldsymbol{S}_{(12) 1}, \quad \widehat{\Delta}_{22}=\frac{N_{1}-1}{N_{1}}\left[\boldsymbol{S}_{1(12), 22}-\boldsymbol{S}_{1(12), 21} \boldsymbol{S}_{1(12), 1}^{-1} \boldsymbol{S}_{1(12), 12}\right] .
\end{aligned}
$$

We note that this statistic was originally obtained by Krishnamoorthy and Pannala (1999). We also note that $Q_{1}$ and $Q_{2}$ are not exactly independent. We propose a test using $R_{2}$, which is independent of $Q_{1}$, instead of $Q_{2}$. We suggest

$$
Q_{M}=Q_{1}+R_{2}
$$

as a new test statistic, where

$$
R_{2}=\frac{Q_{2}}{1+Q_{2 d}}, \quad Q_{2 d}=\frac{N_{1}}{N_{1}-1} \bar{x}_{11}^{\prime} S_{1(12), 11}^{-1} \bar{x}_{11}
$$


The likelihood ratio statistic with two-step monotone missing data using $R_{2}$ is discussed by Krishnamoorthy and Pannala (1998). We can derive an asymptotic expansion of the test statistic more accurately because $Q_{1}$ and $R_{2}$ are exactly independent. Without loss of generality, we may assume that $\boldsymbol{\Sigma}=\boldsymbol{I}=\left(\begin{array}{cc}\boldsymbol{I}_{p_{1}} & \boldsymbol{O} \\ \boldsymbol{O} & \boldsymbol{I}_{p_{2}}\end{array}\right)$.

Initially, we consider a statistic expansion of $Q_{1}$. Let

$$
\overline{\boldsymbol{x}}_{1(12)}=\frac{1}{\sqrt{N_{2}}} z, \quad \boldsymbol{S}_{(12) 1}=\boldsymbol{I}_{p_{1}}+\frac{1}{\sqrt{N_{2}-1}} \boldsymbol{V} .
$$

Yagi et al. (2019) have provided the expansions of $Q_{1}$ and $Q_{2}$. According to their, $Q_{1}$ is expanded as

$$
Q_{1}=z^{\prime} z-\frac{1}{\sqrt{N_{2}}} z^{\prime} V z+\frac{1}{N_{2}}\left(z^{\prime} z+z^{\prime} V^{2} z\right)+O_{p}\left(N_{2}^{-\frac{3}{2}}\right),
$$

and the characteristic function of $Q_{1}$ is

$$
\phi_{Q_{1}}(t)=E\left[\exp \left(i t Q_{1}\right)\right]=u^{-\frac{1}{2} p_{1}}+\frac{1}{N_{1}} \sum_{j=0}^{2} \beta_{j, 1} u^{-\frac{1}{2} p_{1}-j}+O\left(N_{1}^{-2}\right),
$$

where

$$
u=1-2 i t, \beta_{0,1}=-\frac{1}{4} p_{1}\left(p_{1}+2\right), \beta_{1,1}=0, \beta_{2,1}=-\beta_{0,1} .
$$

Similarly, let

$$
\overline{\boldsymbol{x}}_{11}=\frac{1}{\sqrt{N_{1}}} z_{1}, \overline{\boldsymbol{x}}_{12}=\frac{1}{\sqrt{N_{1}}} z_{2}, \boldsymbol{S}_{1(12)}=\left(\begin{array}{cc}
\boldsymbol{I}_{p_{1}} & \boldsymbol{O} \\
\boldsymbol{O} & \boldsymbol{I}_{p_{2}}
\end{array}\right)+\frac{1}{\sqrt{N_{1}-1}}\left(\begin{array}{cc}
\boldsymbol{V}_{11} & \boldsymbol{V}_{12} \\
\boldsymbol{V}_{21} & \boldsymbol{V}_{22}
\end{array}\right)
$$

Then, $Q_{2}$ can be expanded as

$$
Q_{2}=z_{2}^{\prime} z_{2}+\frac{1}{\sqrt{N_{1}}} A_{1}+\frac{1}{N_{1}} A_{2}+O_{p}\left(N_{1}^{-\frac{3}{2}}\right)
$$

where

$$
\begin{aligned}
& A_{1}=-2 z_{2}^{\prime} V_{21} z_{1}-z_{2}^{\prime} V_{22} z_{2}, \\
& A_{2}=2\left(z_{2}^{\prime} V_{21} V_{11} z_{1}+z_{2}^{\prime} V_{22} V_{21} z_{1}\right)+z_{1}^{\prime} V_{12} V_{21} z_{1}+z_{2}^{\prime} z_{2}+z_{2}^{\prime} V_{21} V_{12} z_{2}+z_{2}^{\prime} V_{22}^{2} z_{2} .
\end{aligned}
$$

Therefore, the characteristic function of $Q_{2}$ is

$$
\phi_{Q_{2}}(t)=E\left[\exp \left(i t Q_{2}\right)\right]=u^{-\frac{1}{2} p_{2}}+\frac{1}{N_{1}} \sum_{j=0}^{2} \beta_{j, 2} u^{-\frac{1}{2} p_{2}-j}+O\left(N_{1}^{-2}\right),
$$

where

$$
\beta_{0,2}=-\frac{1}{4} p_{2}\left(4 p_{1}+p_{2}+2\right), \quad \beta_{1,2}=p_{1} p_{2}, \quad \beta_{2,2}=\frac{1}{4} p_{2}\left(p_{2}+2\right) .
$$

Further, we derive the characteristic function of $R_{2}$. The denominator of $R_{2}$ can be expanded as follows:

$$
\left(1+Q_{2 d}\right)^{-1}=1-\frac{1}{N_{1}} z_{1}^{\prime} z_{1}+O_{p}\left(N_{1}^{-\frac{3}{2}}\right)
$$

Then, $R_{2}$ is expanded as

$$
R_{2}=z_{2}^{\prime} z_{2}+\frac{1}{\sqrt{N_{1}}} A_{1}+\frac{1}{N_{1}}\left(A_{2}-z_{1}^{\prime} z_{1} z_{2}^{\prime} z_{2}\right)+O_{p}\left(N_{1}^{-\frac{3}{2}}\right)
$$


This means that an extra term $-\frac{1}{N_{1}}\left(z_{1}^{\prime} z_{1} z_{2}^{\prime} z_{2}\right)$ has been added to the expansion of $Q_{2}$. Therefore, the characteristic function of $R_{2}$ can be expressed as

$$
\begin{aligned}
\phi_{R_{2}}(t) & =E\left[\exp \left(i t R_{2}\right)\right] \\
& =u^{-\frac{1}{2} p_{2}}+\frac{1}{N_{1}}\left[\left(\beta_{0,2}+\frac{1}{2} p_{1} p_{2}\right) u^{-\frac{1}{2} p_{2}}+\left(\beta_{1,2}-\frac{1}{2} p_{1} p_{2}\right) u^{-\frac{1}{2} p_{2}-1}+\beta_{2,2} u^{-\frac{1}{2} p_{2}-2}\right]+O\left(N_{1}^{-\frac{3}{2}}\right) .
\end{aligned}
$$

Then, the characteristic function of $Q_{M}$ is

$$
\phi_{Q_{M}}(t)=\phi_{Q_{1}}(t) \phi_{R_{2}}(t)=u^{-\frac{1}{2} p_{2}}+\frac{1}{N_{1}} \sum_{j=0}^{2} \beta_{j}^{*} u^{-\frac{p}{2}-j}+O\left(N_{1}^{-\frac{3}{2}}\right),
$$

where

$$
\begin{aligned}
& \beta_{0}^{*}=-\frac{1}{4}\left\{\frac{1}{1+r_{2}} p_{1}\left(p_{1}+2\right)+p_{2}\left(2 p_{1}+p_{2}+2\right)\right\}, \quad \beta_{1}^{*}=\frac{1}{2} p_{1} p_{2}, \\
& \beta_{2}^{*}=\frac{1}{4}\left\{\frac{1}{1+r_{2}} p_{1}\left(p_{1}+2\right)+p_{2}\left(p_{2}+2\right)\right\}, \quad r_{2}=\frac{n_{2}}{N_{1}} .
\end{aligned}
$$

Therefore,

$$
\operatorname{Pr}\left(Q_{M} \leq x\right)=G_{p}(x)+\frac{1}{N_{1}} \sum_{j=0}^{2} \beta_{j}^{*} G_{p+2 j}(x)+O\left(N_{1}^{-2}\right),
$$

where $G_{f}(x)$ is the distribution function of $\chi^{2}$-variate with $f$ degrees of freedom. An approximation to the $100 \alpha$ percentile of $Q_{M}$ is given by

$$
q_{M A E}(\alpha)=\chi_{p}^{2}(\alpha)-\frac{1}{N_{1}}\left[\frac{2 \chi_{p}^{2}(\alpha)}{p}\left\{\beta_{0}^{*}-\frac{\beta_{2}^{*}}{p+2} \chi_{p}^{2}(\alpha)\right\}\right],
$$

where $\chi_{p}^{2}(\alpha)$ is the upper $100 \alpha$ percentile of $\chi^{2}$ distribution with $p$ degrees of freedom.

\subsection{Two Sample Problem}

In this section, we consider the two sample case. Let us consider the following hypothesis:

$$
H_{0}: \boldsymbol{\mu}^{(1)}=\boldsymbol{\mu}^{(2)} \text { vs. } H_{1}: \boldsymbol{\mu}^{(1)} \neq \boldsymbol{\mu}^{(2)}
$$

Yu et al. (2006) provided the simplified $T^{2}$ statistic for the two sample case. We express the simplified $T^{2}$ statistic as follows:

$$
Q=Q_{1}+Q_{2}
$$

where

$$
\begin{aligned}
Q_{i} & =\frac{N_{3-i}^{(1)} N_{3-i}^{(2)}}{N_{3-i}^{(1)}+N_{3-i}^{(2)}}\left(\widehat{\boldsymbol{\eta}}_{i}^{(1)}-\widehat{\boldsymbol{\eta}}_{i}^{(2)}\right)^{\prime} \widehat{\Delta}_{i i_{p \ell}}^{-1}\left(\widehat{\boldsymbol{\eta}}_{i}^{(1)}-\widehat{\boldsymbol{\eta}}_{i}^{(2)}\right), i=1,2, \quad N_{i}^{(\ell)}=\sum_{j=1}^{i} n_{j}^{(\ell)}, \\
\widehat{\boldsymbol{\eta}}_{1}^{(\ell)} & =\overline{\boldsymbol{x}}_{(12) 1}^{(\ell)}, \quad \widehat{\boldsymbol{\eta}}_{2}^{(\ell)}=\overline{\boldsymbol{x}}_{12}^{(\ell)}-\sum_{\ell=1}^{2}\left\{\left(N_{1}^{(\ell)}-1\right) \boldsymbol{S}_{1(12), 21}^{(\ell)}\right\} \sum_{\ell=1}^{2}\left\{\left(N_{1}^{(\ell)}-1\right) \boldsymbol{S}_{1(12), 11}^{(\ell)}\right\}^{-1} \overline{\boldsymbol{x}}_{11}^{(\ell)}, \\
\widehat{\Delta}_{11_{p \ell}} & =\frac{1}{N_{2}^{(1)}+N_{2}^{(2)}} \sum_{\ell=1}^{2}\left(N_{2}^{(\ell)}-1\right) \boldsymbol{S}_{(12) 1}^{(\ell)}, \\
\widehat{\Delta}_{22_{p \ell}} & =\frac{1}{N_{1}^{(1)}+N_{1}^{(2)}}\left[\sum_{\ell=1}^{2}\left\{\left(N_{1}^{(\ell)}-1\right) \boldsymbol{S}_{1(12), 22}^{(\ell)}\right\}-\sum_{\ell=1}^{2}\left\{\left(N_{1}^{(\ell)}-1\right) \boldsymbol{S}_{1(12), 21}^{(\ell)}\right\} \sum_{\ell=1}^{2}\left\{\left(N_{1}^{(\ell)}-1\right) \boldsymbol{S}_{1(12), 11}^{(\ell)}\right\}^{-1} \sum_{\ell=1}^{2}\left\{\left(N_{1}^{(\ell)}-1\right) \boldsymbol{S}_{1(12), 12}^{(\ell)}\right\}\right] .
\end{aligned}
$$

Then, we suggest

$$
Q_{M}=Q_{1}+R_{2}
$$


as a new test statistic, where

$$
R_{2}=\frac{Q_{2}}{1+Q_{2 d}}, \quad Q_{2 d}=\frac{N_{1}^{(1)} N_{1}^{(2)}}{N_{1}^{(1)}+N_{1}^{(2)}}\left(\overline{\boldsymbol{x}}_{11}^{(1)}-\overline{\boldsymbol{x}}_{11}^{(2)}\right)^{\prime}\left\{\sum_{\ell=1}^{2}\left(N_{1}^{(\ell)}-1\right) \boldsymbol{S}_{1(12), 11}^{(\ell)}\right\}^{-1}\left(\overline{\boldsymbol{x}}_{11}^{(1)}-\overline{\boldsymbol{x}}_{11}^{(2)}\right)
$$

Without loss of generality, we may assume that $\boldsymbol{\Sigma}=\boldsymbol{I}=\left(\begin{array}{cc}\boldsymbol{I}_{p_{1}} & \boldsymbol{O} \\ \boldsymbol{O} & \boldsymbol{I}_{p_{2}}\end{array}\right)$.

In a derivation that is similar to the one sample case, at the beginning we consider a statistic expansion of $Q_{1}$. Let $\boldsymbol{\mu}^{(1)}=\boldsymbol{\mu}^{(2)}=\boldsymbol{\mu}=\left(\boldsymbol{\mu}_{1}^{\prime}, \boldsymbol{\mu}_{2}^{\prime}\right)^{\prime}$, where $\boldsymbol{\mu}_{i}$ is a $p_{i} \times 1$ vector. Then, let

$$
\overline{\boldsymbol{x}}_{(12) 1}^{(\ell)}=\boldsymbol{\mu}_{1}+\frac{1}{\sqrt{N_{2}^{(\ell)}}} \boldsymbol{u}^{(\ell)}, \boldsymbol{S}_{(12) 1}^{(\ell)}=\boldsymbol{I}_{p_{1}}+\frac{1}{\sqrt{N_{2}^{(\ell)}-1}} \boldsymbol{W}^{(\ell)}, \quad \ell=1,2 .
$$

Yagi et al. (2018) have provided the expansions of $Q_{1}$ and $Q_{2}$. According to their work, $Q_{1}$ is expanded as

$$
Q_{1}=\boldsymbol{u}^{\prime} \boldsymbol{u}-\frac{1}{\sqrt{N_{2}}} \boldsymbol{u}^{\prime} \boldsymbol{W} \boldsymbol{u}+\frac{1}{N_{2}}\left\{\frac{2}{1+t} \boldsymbol{u}^{\prime} \boldsymbol{u}+\boldsymbol{u}^{\prime} \boldsymbol{W}^{2} \boldsymbol{u}\right\}+O_{p}\left(N_{2}^{-\frac{3}{2}}\right),
$$

where

$$
\boldsymbol{u}=\left(\frac{t}{1+t}\right)^{\frac{1}{2}}\left(\boldsymbol{u}^{(1)}-\frac{1}{\sqrt{t}} \boldsymbol{u}^{(2)}\right), \quad \boldsymbol{W}=\frac{1}{1+t} \boldsymbol{W}^{(1)}+\frac{\sqrt{t}}{1+t} \boldsymbol{W}^{(2)}, \quad t=\frac{N_{2}^{(2)}}{N_{2}^{(1)}} .
$$

Then, the characteristic function of $Q_{1}$ can be written as

$$
\phi_{Q_{1}}(t)=u^{-\frac{1}{2} p_{1}}+\frac{1}{N_{2}^{(1)}} \sum_{j=0}^{2} \gamma_{j, 1} u^{-\frac{1}{2} p_{1}-j}+O\left(N_{2}^{(1)^{-2}}\right),
$$

where

$$
\gamma_{0,1}=-\frac{1}{4(1+t)} p_{1}\left(p_{1}+4\right), \quad \gamma_{1,1}=\frac{1}{2(1+t)} p_{1}, \quad \gamma_{2,1}=\frac{1}{4(1+t)} p_{1}\left(p_{1}+2\right) . \quad u=1-2 i t .
$$

Then, let

$$
\begin{aligned}
& \overline{\boldsymbol{x}}_{11}^{(\ell)}=\boldsymbol{\mu}_{1}+\frac{1}{\sqrt{N_{1}^{(\ell)}}} \boldsymbol{u}_{1}^{(\ell)}, \overline{\boldsymbol{x}}_{12}^{(\ell)}=\boldsymbol{\mu}_{2}+\frac{1}{\sqrt{N_{1}^{(\ell)}}} \boldsymbol{u}_{2}^{(\ell)}, \\
& \boldsymbol{S}_{1(12)}^{(\ell)}=\left(\begin{array}{cc}
\boldsymbol{I}_{p_{1}} & \boldsymbol{O} \\
\boldsymbol{O} & \boldsymbol{I}_{p_{2}}
\end{array}\right)+\frac{1}{\sqrt{N_{1}^{(\ell)}-1}}\left(\begin{array}{ll}
\boldsymbol{W}_{11}^{(\ell)} & \boldsymbol{W}_{12}^{(\ell)} \\
\boldsymbol{W}_{21}^{(\ell)} & \boldsymbol{W}_{22}^{(\ell)}
\end{array}\right), \quad \ell=1,2 .
\end{aligned}
$$

Then, $Q_{2}$ is expanded as

$$
Q_{2}=\boldsymbol{u}_{2}^{\prime} \boldsymbol{u}_{2}+\frac{1}{\sqrt{N_{1}^{(1)}}} A_{3}+\frac{1}{N_{1}^{(1)}} A_{4}+O_{p}\left(N_{1}^{(1)^{-\frac{3}{2}}}\right)
$$

where

$$
\begin{aligned}
& A_{3}=-2 \boldsymbol{u}_{2}^{\prime} \boldsymbol{W}_{21} \boldsymbol{u}_{1}-\boldsymbol{u}_{2}^{\prime} \boldsymbol{W}_{22} \boldsymbol{u}_{2}, \\
& A_{4}=2\left(\boldsymbol{u}_{2}^{\prime} \boldsymbol{W}_{21} \boldsymbol{W}_{11} \boldsymbol{u}_{1}+\boldsymbol{u}_{2}^{\prime} \boldsymbol{W}_{21}^{2} \boldsymbol{u}_{1}\right)+\boldsymbol{u}_{1}^{\prime} \boldsymbol{W}_{12} \boldsymbol{W}_{21} \boldsymbol{u}_{1}+\boldsymbol{u}_{2}^{\prime} \boldsymbol{W}_{21} \boldsymbol{W}_{12} \boldsymbol{u}_{2}+\frac{2}{1+q} \boldsymbol{u}_{2}^{\prime} \boldsymbol{u}_{2}+\boldsymbol{u}_{2}^{\prime} \boldsymbol{W}_{22}^{2} \boldsymbol{u}_{2}, \\
& \boldsymbol{u}_{1}=\left(\frac{q}{1+q}\right)^{\frac{1}{2}}\left(\boldsymbol{u}_{1}^{(1)}-\frac{1}{\sqrt{q}} \boldsymbol{u}_{1}^{(2)}\right), \quad \boldsymbol{u}_{2}=\left(\frac{q}{1+q}\right)^{\frac{1}{2}}\left(\boldsymbol{u}_{2}^{(1)}-\frac{1}{\sqrt{q}} \boldsymbol{u}_{2}^{(2)}\right), \\
& \left(\begin{array}{ll}
\boldsymbol{W}_{11} & \boldsymbol{W}_{12} \\
\boldsymbol{W}_{21} & \boldsymbol{W}_{22}
\end{array}\right)=\frac{1}{1+q}\left(\begin{array}{ll}
\boldsymbol{W}_{11}^{(1)} & \boldsymbol{W}_{12}^{(1)} \\
\boldsymbol{W}_{21}^{(1)} & \boldsymbol{W}_{22}^{(1)}
\end{array}\right)+\frac{\sqrt{q}}{1+q}\left(\begin{array}{ll}
\boldsymbol{W}_{11}^{(2)} & \boldsymbol{W}_{12}^{(2)} \\
\boldsymbol{W}_{21}^{(2)} & \boldsymbol{W}_{22}^{(2)}
\end{array}\right), \quad q=\frac{N_{1}^{(2)}}{N_{1}^{(1)}} .
\end{aligned}
$$


Therefore, the characteristic function of $Q_{2}$ is

$$
\phi_{Q_{2}}(t)=u^{-\frac{1}{2} p_{2}}+\frac{1}{N_{1}^{(1)}} \sum_{j=0}^{2} \gamma_{j, 2} u^{-\frac{1}{2} p_{1}-j}+O\left(N_{1}^{(1)^{-2}}\right)
$$

where

$$
\gamma_{0,2}=\frac{1}{4(1+q)} p_{2}\left(4 p_{1}+p_{2}+4\right), \quad \gamma_{1,2}=\frac{1}{2(1+q)} p_{2}\left(2 p_{1}+1\right), \quad \gamma_{2,2}=\frac{1}{4(1+q)} p_{2}\left(p_{2}+2\right)
$$

Further, we derive the characteristic function of $R_{2}$. The denominator of $R_{2}$ can be expanded as follows:

$$
\left(1+Q_{2 d}\right)^{-1}=1-\frac{1}{N_{1}^{(1)}} \times \frac{q}{(1+q)^{2}}\left(\boldsymbol{u}_{1}^{(1)}-\frac{1}{\sqrt{q}} \boldsymbol{u}_{1}^{(2)}\right)^{\prime}\left(\boldsymbol{u}_{1}^{(1)}-\frac{1}{\sqrt{q}} \boldsymbol{u}_{1}^{(2)}\right)+O\left(N_{1}^{(1)^{-\frac{3}{2}}}\right)
$$

Therefore,

$$
R_{2}=\boldsymbol{u}_{2}^{\prime} \boldsymbol{u}_{2}+\frac{1}{\sqrt{N_{1}^{(1)}}} A_{3}+\frac{1}{N_{1}^{(1)}}\left(A_{4}-\frac{1}{1+q} \boldsymbol{u}_{2}^{\prime} \boldsymbol{u}_{2} \boldsymbol{u}_{1}^{\prime} \boldsymbol{u}_{1}\right)+O\left(N_{1}^{(1)^{-\frac{3}{2}}}\right) .
$$

The characteristic function of $R_{2}$ is

$$
\begin{aligned}
\phi_{R_{2}}(t) & =E\left[\exp \left(i t R_{2}\right)\right] \\
& =u^{-\frac{1}{2} p_{2}}+\frac{1}{N_{1}} \sum_{j=0}^{2} \gamma_{j, 3} u^{-\frac{1}{2} p_{2}-j}+\left(N_{1}^{(1)^{-2}}\right),
\end{aligned}
$$

where,

$$
\gamma_{0,3}=-\frac{1}{4(1+q)} p_{2}\left(2 p_{1}+p_{2}+4\right), \quad \gamma_{1,3}=\frac{1}{2(1+q)} p_{2}\left(p_{1}+1\right), \quad \gamma_{2,3}=\frac{1}{4(1+q)} p_{2}\left(p_{2}+2\right) .
$$

Conforming the order of $Q_{1}$ and $R_{2}$ to $v_{1}$,

$$
\begin{aligned}
\phi_{Q_{1}}(t) & =u^{-\frac{1}{2} p_{1}}+\frac{1}{v_{1}}\left\{-\frac{1}{4(1+s)} p_{1}\left(p_{1}+4\right) u^{-\frac{1}{2} p_{1}}\right. \\
& \left.+\frac{1}{2(1+s)} p_{1} u^{-\frac{1}{2} p_{1}-1}+\frac{1}{4(1+s)} p_{1}\left(p_{1}+2\right) u^{-\frac{1}{2} p_{1}-2}\right\}+O\left(v_{1}^{-2}\right) \\
\phi_{R_{2}}(t) & =u^{-\frac{1}{2} p_{2}}+\frac{1}{v_{1}}\left\{-\frac{1}{4} p_{2}\left(p_{2}+2 p_{1}+4\right) u^{-\frac{1}{2} p_{2}}\right. \\
& \left.+\frac{1}{2} p_{2}\left(p_{1}+1\right) u^{-\frac{1}{2} p_{2}-1}+\frac{1}{4} p_{2}\left(p_{2}+2\right) u^{-\frac{1}{2} p_{2}-2}\right\}+O\left(v_{1}^{-2}\right)
\end{aligned}
$$

Since $Q_{M}=Q_{1}+R_{2}$, the characteristic function of $Q_{M}$ is

$$
\begin{aligned}
\phi_{Q_{M}}(t) & =\phi_{Q_{1}}(t) \phi_{R_{2}}(t) \\
& =u^{-\frac{1}{2} p}+\frac{1}{v_{1}} \sum_{j=0}^{2} \gamma_{j}^{*} u^{-\frac{1}{2} p-j}+O\left(v_{1}^{-2}\right),
\end{aligned}
$$

where

$$
\begin{aligned}
& \gamma_{0}^{*}=-\frac{1}{4} p_{2}\left(2 p_{1}+p_{2}+4\right)-\frac{1}{4(1+s)} p_{1}\left(p_{1}+4\right), \gamma_{1}^{*}=\frac{1}{2} p_{2}\left(p_{1}+1\right)+\frac{1}{2(1+s)} p_{1}, \\
& \gamma_{2}^{*}=\frac{1}{4} p_{2}\left(p_{2}+2\right)+\frac{1}{4(1+s)} p_{1}\left(p_{1}+2\right), \quad v_{i}=n_{i}^{(1)}+n_{i}^{(2)}(i=1,2), \quad s=\frac{v_{2}}{v_{1}} .
\end{aligned}
$$

Therefore,

$$
\operatorname{Pr}\left(Q_{M} \leq x\right)=G_{p}(x)+\frac{1}{v_{1}} \sum_{j=0}^{2} \gamma_{j}^{*} G_{p+2 j}(x)+O\left(v_{1}^{-2}\right)
$$


where $G_{f}(x)$ is the distribution function of $\chi^{2}$-variate with $f$ degrees of freedom. An approximation to the $100 \alpha$ percentile of $Q_{M}$ is given by

$$
q_{M}(\alpha)=\chi_{p}^{2}(\alpha)-\frac{1}{v_{1}}\left[\frac{2 \chi_{p}^{2}(\alpha)}{p}\left\{\gamma_{0}^{*}-\frac{\gamma_{2}^{*}}{p+2} \chi_{p}^{2}(\alpha)\right\}\right] .
$$

\section{Transformed Test Statistics}

\subsection{One Sample Case}

The statistics $Q_{1}$ and $Q_{2}$ that are transformed by the Bartlett collection are given by

$$
Q_{1}^{*}=\left(1-\frac{1}{N_{2}}\left(p_{1}+2\right)\right) Q_{1}, \quad R_{2}^{*}=\left(1-\frac{1}{N_{1}}\left(p_{1}+p_{2}+2\right)\right) R_{2} .
$$

Therefore, we suggest the transformed test statistic of $Q_{M}$ as

$$
Q_{M}^{*}=Q_{1}^{*}+R_{2}^{*}
$$

Moreover, Fujikoshi (2000) suggested the Bartlett-type correction for general test statistics. Using this method, the Bartlett-type corrections for $Q_{1}$ and $R_{2}$ are

$$
Y_{1}=\left\{N_{2}-\frac{1}{2}\left(p_{1}+2\right)\right\} \log \left(1+\frac{1}{N_{2}} Q_{1}\right), \text { for } N_{2}-\frac{1}{2}\left(p_{1}+2\right)>0
$$

and

$$
Y_{2 M}=\left\{N_{1}-\frac{1}{2}\left(2 p_{1}+p_{2}+2\right)\right\} \log \left(1+\frac{1}{N_{1}} R_{2}\right), \quad \text { for } N_{1}-\frac{1}{2}\left(2 p_{1}+p_{2}+2\right)>0,
$$

respectively. Then, we suggest the transformed statistic of $Q_{M}$ by Bartlett-type correction as

$$
Y_{M}=Y_{1}+Y_{2 M}
$$

We also have the Bartlett correction of $Q_{M}$ as

$$
Q_{M}^{\dagger}=\left(1+\frac{1}{N_{1}} c_{1}\right) Q_{M},
$$

where

$$
c_{1}=\frac{1}{p}\left\{\frac{1}{1+t} p_{1}\left(p_{1}+2\right)+p_{2}(p+2)\right\} .
$$

Using the result of Fujikoshi (2000), we can obtain the Bartlett-type correction as

$$
Y_{M}^{\dagger}=\left(N_{1} a+b\right) \log \left(1+\frac{1}{N_{1} a} Q_{M}\right), \text { for } a>0 \text { and } N_{1} a+b>0,
$$

where

$$
a=p(p+2)\left\{\frac{1}{1+t} p_{1}\left(p_{1}+2\right)+p_{2} p_{1}+2\right\}^{-1}, \quad b=-\frac{a}{2 p}\left\{\frac{1}{1+t} p_{1}\left(p_{1}+2\right)+p_{2}\left(2 p_{1}+p_{2}+2\right)\right\} .
$$

Krishnarmoorthy and Pannala (1999) proposed approximated upper percentiles of $Q$ distributions using $F$ distribution. Applying its approximation to $Q_{M}$, we propose $q_{M K P}(\alpha)$ as

$$
q_{M K P}(\alpha)=d_{1} F_{p, v_{\mathrm{MKP}}}(\alpha), \quad \text { for } N_{1}>p+4,
$$


where

$$
\begin{aligned}
d_{1} & =M_{1} \frac{v_{\mathrm{KP}}-2}{v_{\mathrm{KP}}}, v_{\mathrm{KP}}=\frac{4 p M_{2}-2(p+2) M_{1}^{2}}{p M_{2}-(p+2) M_{1}^{2}}, \\
M_{1} & =E\left(Q_{M}\right)=E\left(Q_{1}\right)+E\left(R_{2}\right) \\
& =\frac{N_{2} p_{1}}{N_{2}-p_{1}-2}+\frac{N_{1} p_{2}}{N_{1}-p-2}, \\
M_{2} & =E\left(Q_{M}^{2}\right) \\
& =E\left(Q_{1}^{2}\right)+2 E\left(Q_{1}\right) E\left(R_{2}\right)+E\left(R_{2}^{2}\right), \\
E\left(Q_{1}^{2}\right) & =\frac{N_{2}^{2} p_{1}\left(p_{1}+2\right)}{\left(N_{2}-p_{1}-2\right)\left(N_{2}-p_{1}-4\right)}, \quad E\left(R_{2}^{2}\right)=\frac{N_{1}^{2} p_{2}\left(p_{2}+2\right)}{\left(N_{1}-p-2\right)\left(N_{1}-p-4\right)} .
\end{aligned}
$$

This approximation $q_{M K P}(\alpha)$ is closer to the truth than the approximation for $Q$ that Krishnarmoorthy and Pannala (1999) originally proposed because $Q_{1}$ and $R_{2}$ are independent, although $Q_{1}$ and $Q_{2}$ are not independent.

\subsection{Two Sample Problem}

We suggest the transformed test statistics similar to that of the one sample case. The statistic $Q_{1}$ and $Q_{2}$ that are transformed by the Bartlett collection are given by

$$
Q_{1}^{*}=\left(1-\frac{1}{v_{1}+v_{2}}\left(p_{1}+3\right)\right) Q_{1}, \quad R_{2}^{*}=\left(1-\frac{1}{v_{1}}\left(p_{1}+p_{2}+3\right)\right) R_{2} .
$$

Therefore, we suggest the transformed test statistic of $Q_{M}$ as

$$
Q_{M}^{*}=Q_{1}^{*}+R_{2}^{*}
$$

Moreover, by applying the Bartlett-type correction of $Q_{1}$ and $R_{2}$, we obtain the following:

$$
Y_{1}=\left\{\left(v_{1}+v_{2}\right) a_{Y_{1}}+b_{Y_{1}}\right\} \log \left(1+\frac{1}{\left(v_{1}+v_{2}\right) a_{Y_{1}}} Q_{1}\right), \quad \text { for }\left(v_{1}+v_{2}\right) a_{Y_{1}}+b_{Y_{1}}>0,
$$

where

$$
a_{Y_{1}}=1+s, \quad b_{Y_{1}}=-\frac{1}{2}\left(p_{1}+4\right),
$$

and

$$
Y_{2 M}=\left\{v_{1}+-\frac{1}{2}\left(2 p_{1}+p_{2}+4\right)\right\} \log \left(1+\frac{1}{v_{1}} R_{2}\right), \quad \text { for } v_{1}+-\frac{1}{2}\left(2 p_{1}+p_{2}+4\right)>0 .
$$

Then, we suggest the transformed statistic of $Q_{M}$ by the Bartlett-type correction as

$$
Y_{M}=Y_{1}+Y_{2 M} .
$$

We have the Bartlett correction of $Q_{M}$ as

$$
Q_{M}^{\dagger}=\left(1+\frac{1}{v_{1}} c_{1}\right) Q_{M},
$$

where

$$
c_{1}=\frac{1}{p}\left\{\frac{1}{1+s} p_{1}\left(p_{1}+3\right)+p_{2}\left(p_{1}+p_{2}+3\right)\right\} .
$$

In addition, using the result of Fujikoshi (2000), we can obtain the Bartlett-type correction as

$$
Y_{M}^{\dagger}=\left(v_{1} a+b\right) \log \left(1+\frac{1}{v_{1} a} Q_{M}\right), \text { for } a>0, N_{1} a+b>0,
$$


where

$$
a=p(p+2)\left\{\frac{1}{1+s} p_{1}\left(p_{1}+2\right)+p_{2}\left(p_{2}+2\right)\right\}^{-1}, \quad b=-\frac{a}{2 p}\left\{-\frac{1}{1+s} p_{1}\left(p_{1}+4\right)+p_{2}\left(-2 p+p_{2}-4\right)\right\} .
$$

Applying the approximation proposed by Yu et al. to $Q_{M}$, we propose $q_{M Y K P}(\alpha)$ as

$$
q_{M Y K P}(\alpha)=d_{2} F_{p, V_{Y K P}}(\alpha), \quad \text { for } N_{1}^{(1)}+N_{1}^{(2)}>p+5,
$$

where

$$
\begin{aligned}
d_{2} & =G_{1} \frac{v_{\mathrm{YKP}}-2}{v_{\mathrm{YKP}}}, \quad v_{\mathrm{YKP}}=\frac{4 p G_{2}-2(p+2) G_{1}^{2}}{p G_{2}-(p+2) G_{1}^{2}}, \\
G_{1} & =E\left(Q_{M}\right)=E\left(Q_{1}\right)+E\left(R_{2}\right) \\
& =\frac{\left(N_{2}^{(1)}+N_{2}^{(2)}\right) p_{1}}{N_{2}^{(1)}+N_{2}^{(2)}-p_{1}-3}+\frac{\left(n_{1}^{(1)}+n_{1}^{(2)}\right) p_{2}}{n_{1}^{(1)}+n_{1}^{(2)}-p-3}, \\
G_{2} & =E\left(Q_{M}^{2}\right) \\
& =E\left(Q_{1}^{2}\right)+2 E\left(Q_{1}\right) E\left(R_{2}\right)+E\left(R_{2}^{2}\right), \\
E\left(Q_{1}^{2}\right) & =\frac{\left(N_{2}^{(1)}+N_{2}^{(2)}\right)^{2} p_{1}\left(p_{1}+2\right)}{\left(N_{2}^{(1)}+N_{2}^{(2)}-p_{1}-3\right)\left(N_{2}^{(1)}+N_{2}^{(2)}-p_{1}-5\right)}, \quad E\left(R_{2}^{2}\right)=\frac{\left(N_{1}^{(1)}+N_{1}^{(2)}\right)^{2} p_{2}\left(p_{2}+2\right)}{\left(N_{1}^{(1)}+N_{1}^{(2)}-p-3\right)\left(N_{1}^{(1)}+N_{1}^{(2)}-p-5\right)} .
\end{aligned}
$$

\section{Numerical Simulations}

In this section, we describe the accuracy of the approximations and the asymptotic behavior of approximate upper percentiles of the test statistic $Q_{M}$ for the one sample and two sample problems. We compare the approximate upper percentiles of $Q_{M}$, some of the transformed test statistics proposed in Section 4, and the test using Krishnamoorthy and Pannala (1999) and Yu et al. (2006)'s approximation. Further, we compare the test $Q_{M}$ we proposed with test $Q$. Their upper $100 \alpha$ percentiles and Type I errors are defined as the following:

1. $q_{M}$ and $\alpha_{\chi_{M}^{2}}=100 \operatorname{Pr}\left(Q_{M}>\chi_{p}^{2}(\alpha)\right)$ : for test $Q_{M}$ in (3) and (6)

2. $q_{M A E}$ and $\alpha_{M A E}=100 \operatorname{Pr}\left(Q_{M}>q_{M A E}(\alpha)\right)$ : for asymptotic expansion approximation (MAE) test in (4) and (7)

3. (a) $q_{M K P}$ and $\alpha_{M K P}=100 \operatorname{Pr}\left(Q_{M}>q_{M K P}(\alpha)\right)$

: for applying Krishnamoorthy and Pannala's approximation to $Q_{M}$ in (12) (one sample)

(b) $q_{M Y K P}$ and $\alpha_{M Y K P}=100 \operatorname{Pr}\left(Q_{M}>q_{M Y K P}(\alpha)\right)$

: for applying Yu, Krishnamoorthy and Pannala's approximation to $Q_{M}$ in (17) (two sample)

4. $q_{Q_{M}^{*}}$ and $\alpha_{Q_{M}^{*}}=100 \operatorname{Pr}\left(Q_{M}^{*}>\chi_{p}^{2}(\alpha)\right)$ : for test $Q_{M}^{*}$ with Bartlett correction in (8) and (13)

5. $q_{Q_{M}^{\dagger}}$ and $\alpha_{Q_{M}^{\dagger}}=100 \operatorname{Pr}\left(Q_{M}^{\dagger}>\chi_{p}^{2}(\alpha)\right)$ : for test $Q_{M}^{\dagger}$ with Bartlett correction in (10) and (15)

6. $q_{Y_{M}}$ and $\alpha_{Y_{M}}=100 \operatorname{Pr}\left(Y_{M}>\chi_{p}^{2}(\alpha)\right)$ : for test $Y_{M}$ based on Bartlett-type correction in (9) and (14)

7. $q_{Y_{M}^{\dagger}}$ and $\alpha_{Y_{M}^{\dagger}}=100 \operatorname{Pr}\left(Y_{M}^{\dagger}>\chi_{p}^{2}(\alpha)\right)$ : for test $Y_{M}^{\dagger}$ with Bartlett-type correction in (11) and (16)

8. $q$ and $\alpha_{\chi^{2}}=100 \operatorname{Pr}\left(Q>\chi_{p}^{2}(\alpha)\right)$ : for test $Q$

9. $q_{A E}$ and $\alpha_{A E}=100 \operatorname{Pr}\left(Q>q_{A E}(\alpha)\right)$ : for asymptotic expansion approximation (AE) test

10. (a) $q_{K P}$ and $\alpha_{K P}=100 \operatorname{Pr}\left(Q>q_{K P}(\alpha)\right)$

: for applying Krishnamoorthy and Pannala's approximation to $Q$ (one sample)

(b) $q_{Y K P}$ and $\alpha_{Y K P}=100 \operatorname{Pr}\left(Q>q_{Y K P}(\alpha)\right)$

: for applying Yu, Krishnamoorthy and Pannala's approximation to $Q$ (two sample)

11. $q_{Q^{*}}$ and $\alpha_{Q^{*}}=100 \operatorname{Pr}\left(Q^{*}>\chi_{p}^{2}(\alpha)\right)$ : for test $Q^{*}$ with Bartlett correction 
12. $q_{Q^{\dagger}}$ and $\alpha_{Q^{\dagger}}=100 \operatorname{Pr}\left(Q^{\dagger}>\chi_{p}^{2}(\alpha)\right)$ : for test $Q^{\dagger}$ with Bartlett correction

13. $q_{Y}$ and $\alpha_{Y}=100 \operatorname{Pr}\left(Y>\chi_{p}^{2}(\alpha)\right)$ : for test $Y_{M}$ based on Bartlett-type correction

14. $q_{Y^{\ddagger}}$ and $\alpha_{Y^{\dagger}}=100 \operatorname{Pr}\left(Y^{\dagger}>\chi_{p}^{2}(\alpha)\right)$ : for test $Y^{\dagger}$ with Bartlett-type correction

We note that $1-7$ are about test $Q_{M}$, and $8-14$ are about test $Q$. A Monte Carlo simulation $\left(10^{6}\right)$ was conducted, considering a significance level $\alpha=0.05$. The settings of the parameters $p\left(=p_{1}+p_{2}\right)$, and sample size for the simulation are as follows:

$$
\begin{aligned}
& \text { Case } 1\left(p_{1}, p_{2}\right)=(2,2),(4,4) \\
& \text { (I) }\left(n_{1}, n_{2}\right)=(m, m),\left(m, \frac{m}{2}\right),(m, 2 m), m=10,20,30,40,50,100,200,400 \\
& \text { (II) }\left(n_{1}, n_{2}\right)=(m, 10),(m, 20),(m, 50),(m, 100), m=10,20,30,50,100 \\
& \text { Case } 2 p_{1}=2,4,6,8,10, p_{2}=2: \text { fix, } n_{1}=n_{2}=30,
\end{aligned}
$$

where, in the two sample case, we set $n_{1}=n_{1}^{(1)}=n_{1}^{(2)}, n_{2}=n_{2}^{(1)}=n_{2}^{(2)}$. Tables 1-4 list the simulation results for (I). Tables 5-8 list the for (II), which are obtained by changing the sample size of the complete data $\left(n_{1}\right)$ to a larger value and fixing the sample size of missing data $\left(n_{2}\right)$. Tables 9 and 10 list the simulation results for Case 2, which are obtained increasing the dimension of $p_{1}$ and fixing the missing data of $p_{2}$. Tables $3,4,7,8$, and 10 are for the two sample case. The upper percentiles of the test statistics are closer to the upper percentiles of $\chi^{2}$ distribution when $n_{1}$ is large. Comparing $q_{M A E}$ and $q_{A E}, q_{M A E}$ is a better approximation to the true value $q_{M}$. In particular, the transformed test statistics were observed to have a faster convergence. Comparing the type I error, $\alpha_{M K P}$ and $\alpha_{M Y K P}$ values are approximately 5.00 when the sample size is large. $\alpha_{Y_{M}}$ and $\alpha_{Y_{u}^{\dagger}}$ are always stable (almost 5.00 or more) for any sample size although we find that the type I error using $q_{Y_{M}}$ gose away from 5.00 for large $p$, as shown in Tables 9 and 10. Judging in this light, the result using $q_{M K P}$ and $q_{M Y K P}$ is good approximation even when $p$ is large. Additionally, $q_{Y_{M}^{\dagger}}$ was observed to be more conservative than $q_{M K P}$.

\section{Concluding Remarks}

We considered testing for equality of one and two mean vectors when the observations have two-step monotone missing data. In this case, $T^{2}$-type statistic can be decomposed into sum forms that can be provided asymptotic expansion has been discussed in previous study. However, these are not independent. In this paper, we proposed new test statistics that can be expressed as sum of statistics that are independent. We also considered transformed test statistics that convergence quickly. Further, we performed a Monte Carlo simulation and confirmed that test statistics we proposed showed better $\chi^{2}$ approximation than original test statistics. Finally, we compared some procedures and confirmed that $q_{M K P}(\alpha)$ in (12) and $q_{M Y K P}(\alpha)$ in (17) are good approximations when the sample size of the complete data is large. Additionally, $Y_{M}^{\dagger}$ is a good approximation when considering the approximation accuracy and conservativeness. We recommend using the test statistics properly according to sample size and dimension. We are currently working on this problem with $k$-step $(k \geq 3)$ monotone missing data. 
Table 1. The upper percentiles of test statistics and empirical Type I errors for the one sample case, $\left(p_{1}, p_{2}\right)=(2,2)$

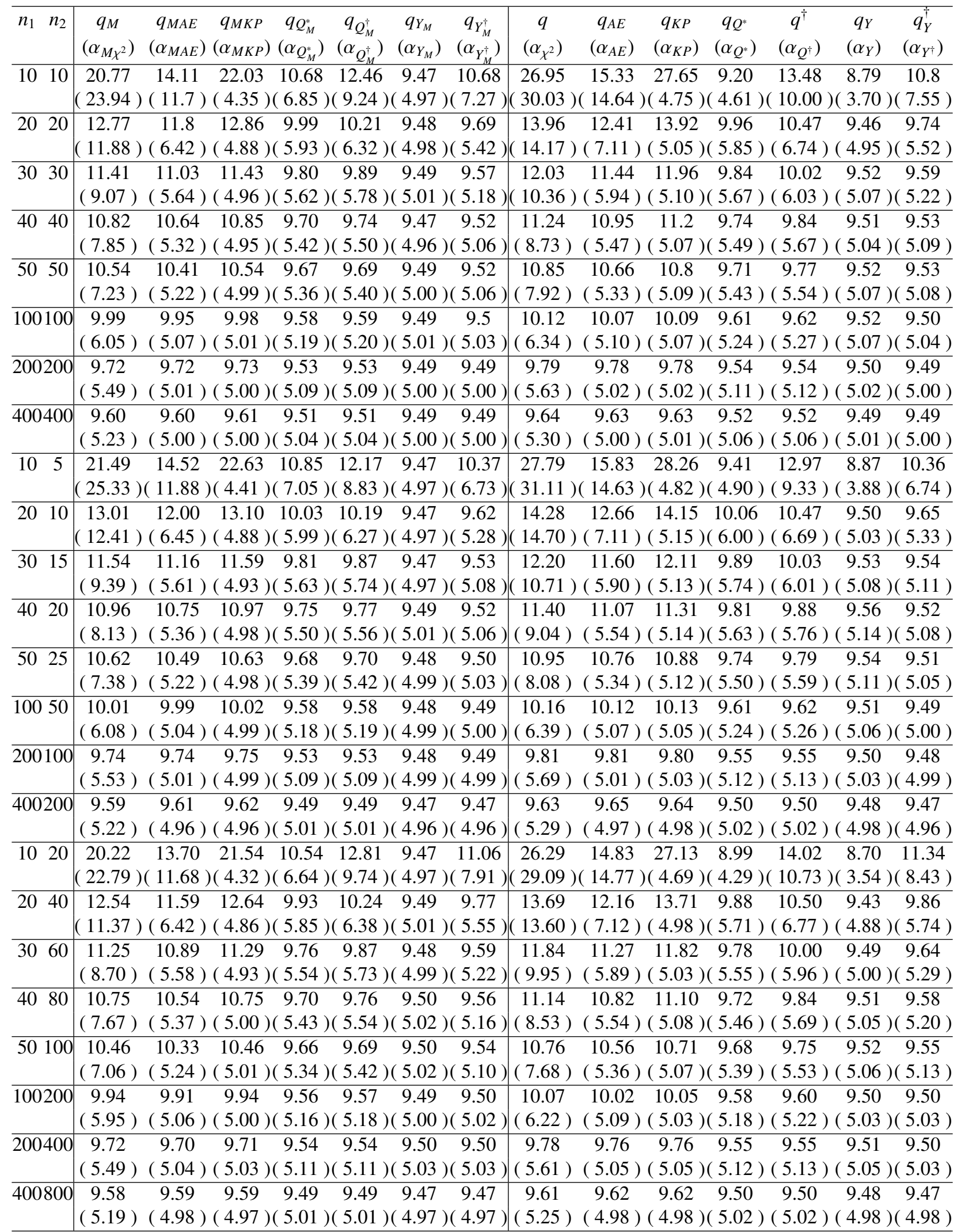

Note: $\chi_{4}^{2}(0.05)=9.49$ 
Table 2. The upper percentiles of test statistics and empirical Type I errors for one the sample case, $\left(p_{1}, p_{2}\right)=(4,4)$

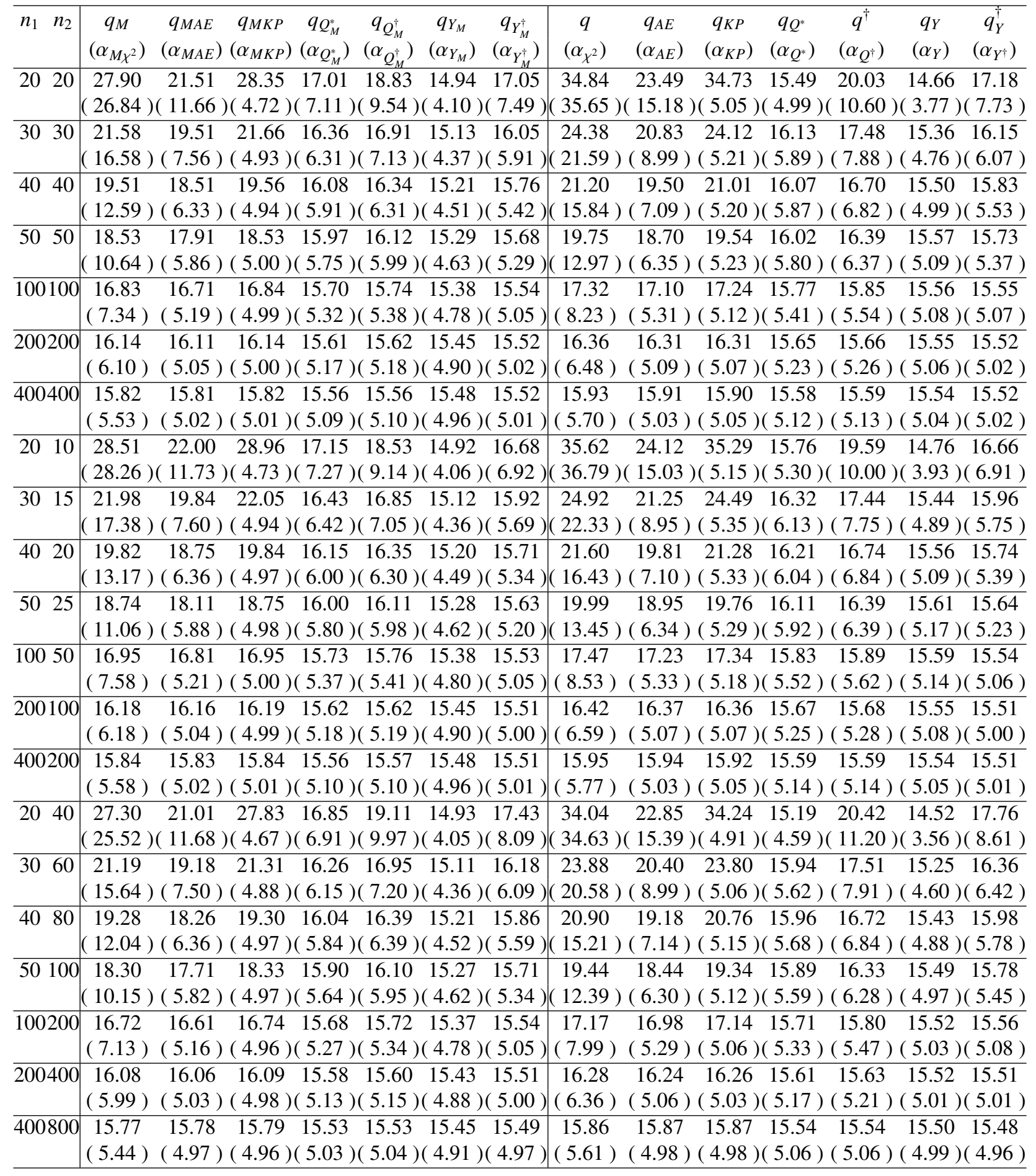

Note: $\chi_{8}^{2}(0.05)=15.51$ 
Table 3. The upper percentiles of test statistics and empirical Type I errors for two sample $\left(p_{1}, p_{2}\right)=(2,2)$

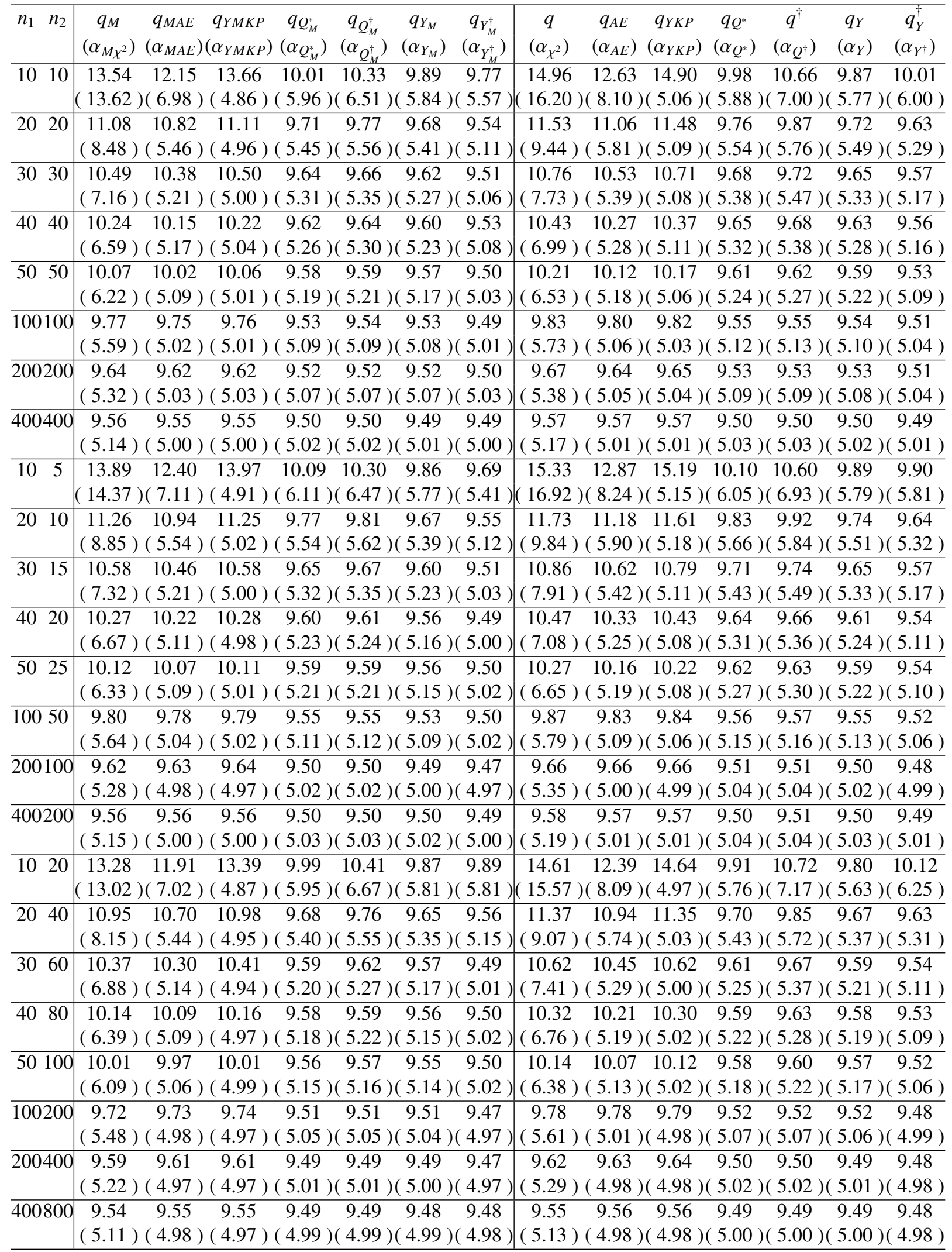

Note: $\chi_{8}^{2}(0.05)=9.49$ 
Table 4. The upper percentiles of test statistics and empirical Type I errors for the two sample case, $\left(p_{1}, p_{2}\right)=(4,4)$

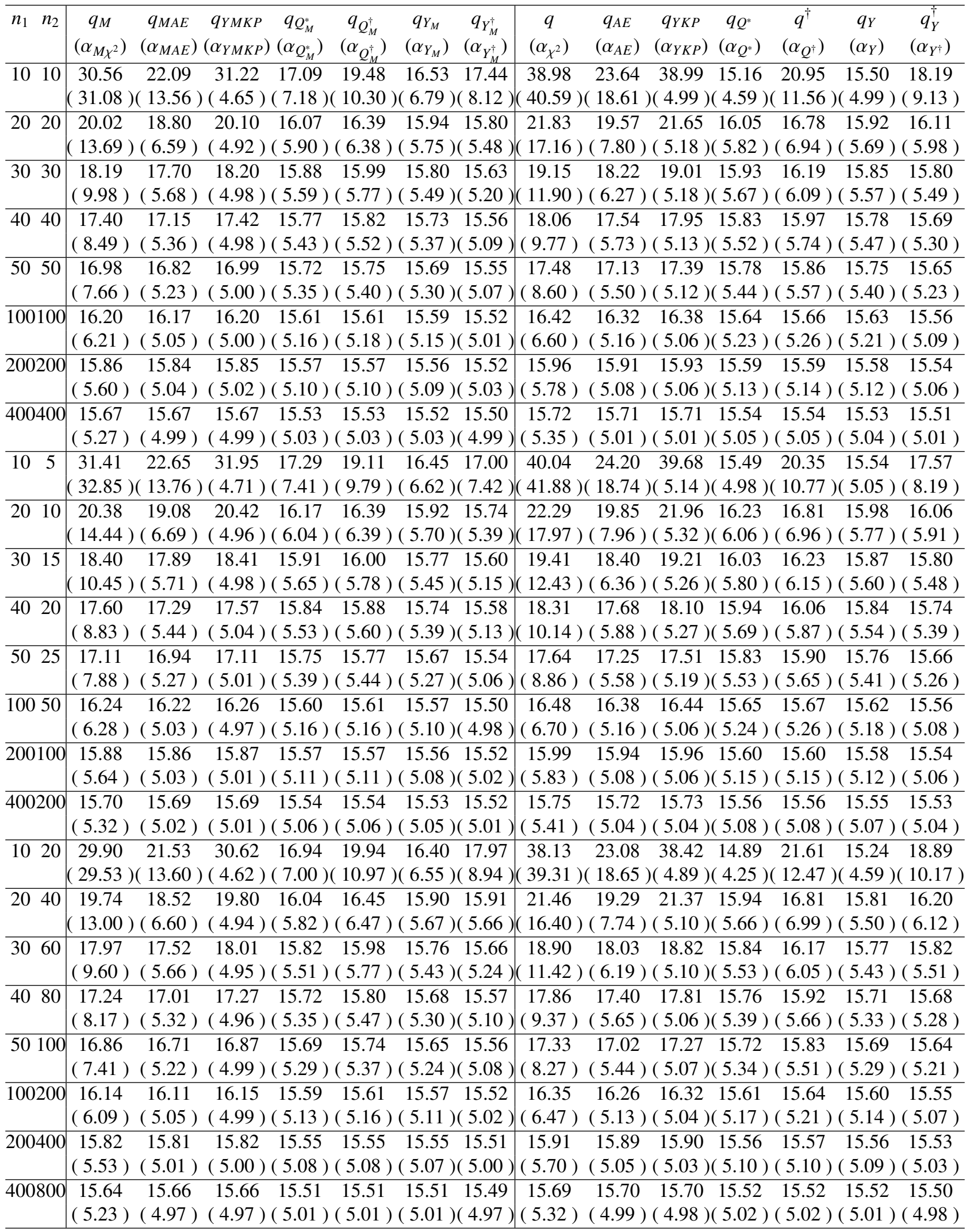

Note: $\chi_{8}^{2}(0.05)=15.51$ 
Table 5. The upper percentiles of test statistics and empirical Type I errors for the one sample case, $\left(p_{1}, p_{2}\right)=(2,2), n_{2}$ : fix

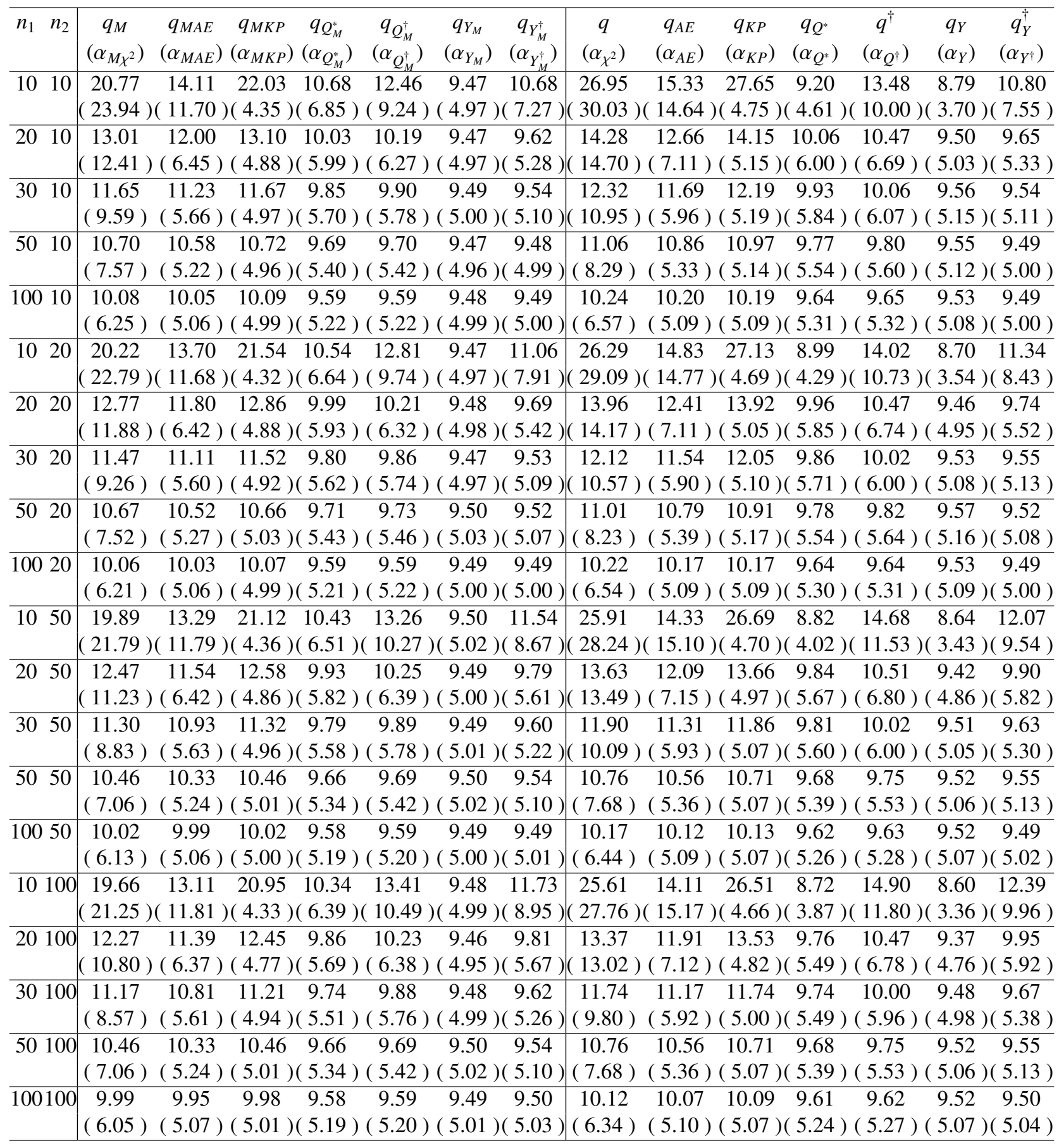

Note: $\chi_{4}^{2}(0.05)=9.49$ 
Table 6. The upper percentiles of test statistics and empirical Type I errors for the one sample case, $\left(p_{1}, p_{2}\right)=(4,4), n_{2}$ : fix

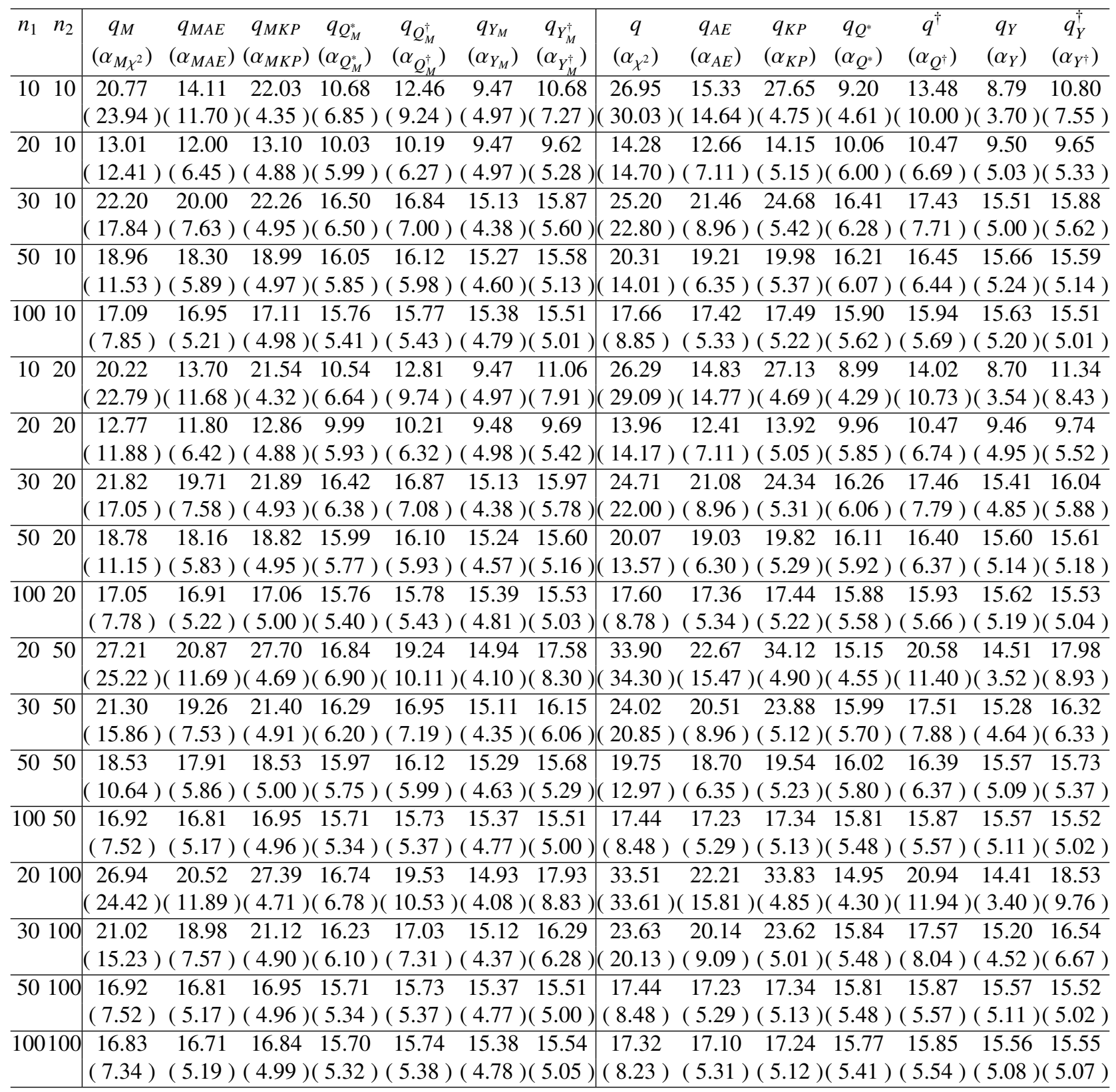

Note: $\chi_{8}^{2}(0.05)=15.51$ 
Table 7. The upper percentiles of test statistics and empirical Type I errors for the two sample case, $\left(p_{1}, p_{2}\right)=(2,2), n_{2}$ : fix

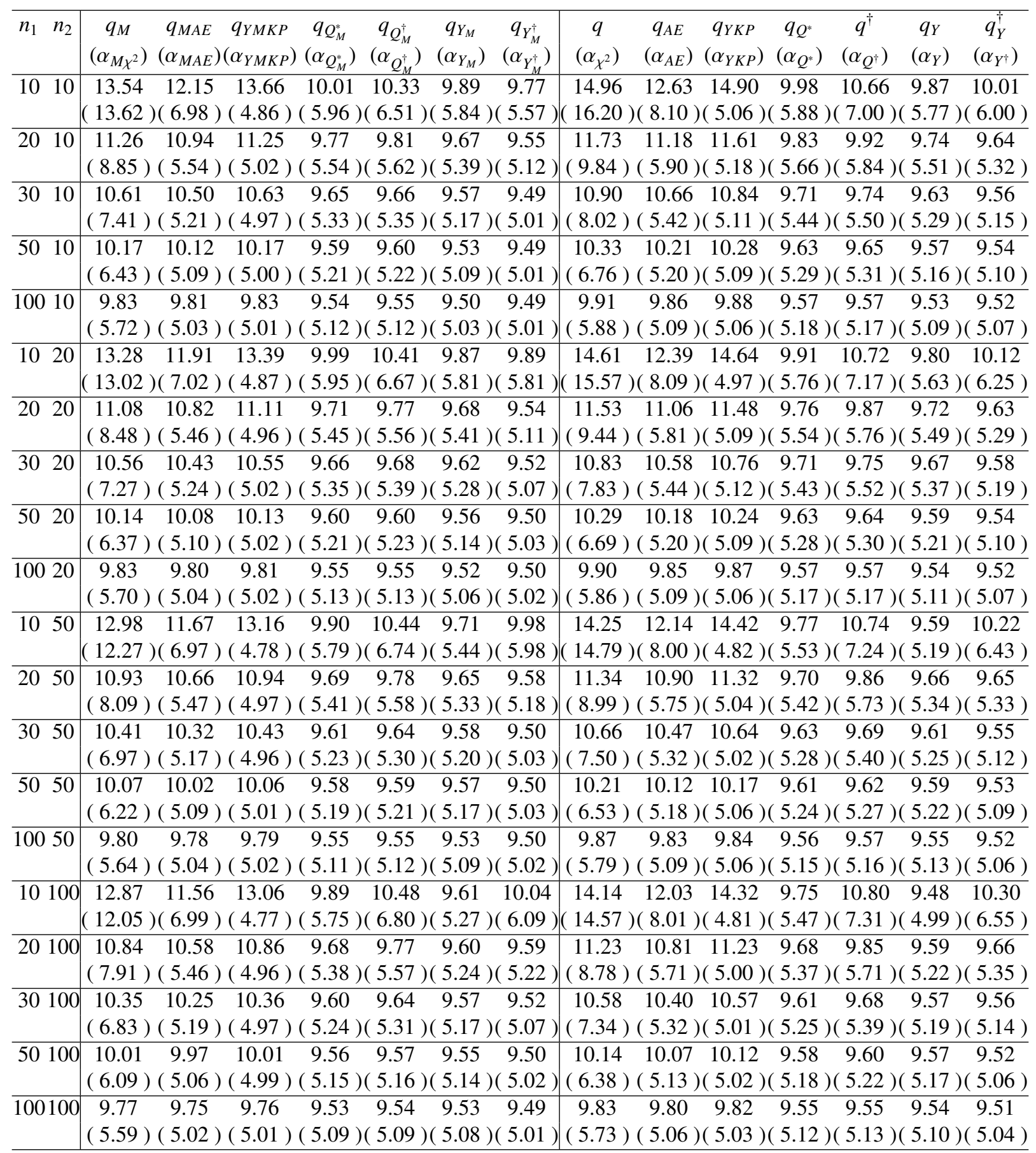

Note: $\chi_{4}^{2}(0.05)=9.49$ 
Table 8. The upper percentiles of test statistics and empirical Type I errors for the two sample case, $\left(p_{1}, p_{2}\right)=(4,4), n_{2}$ : fix

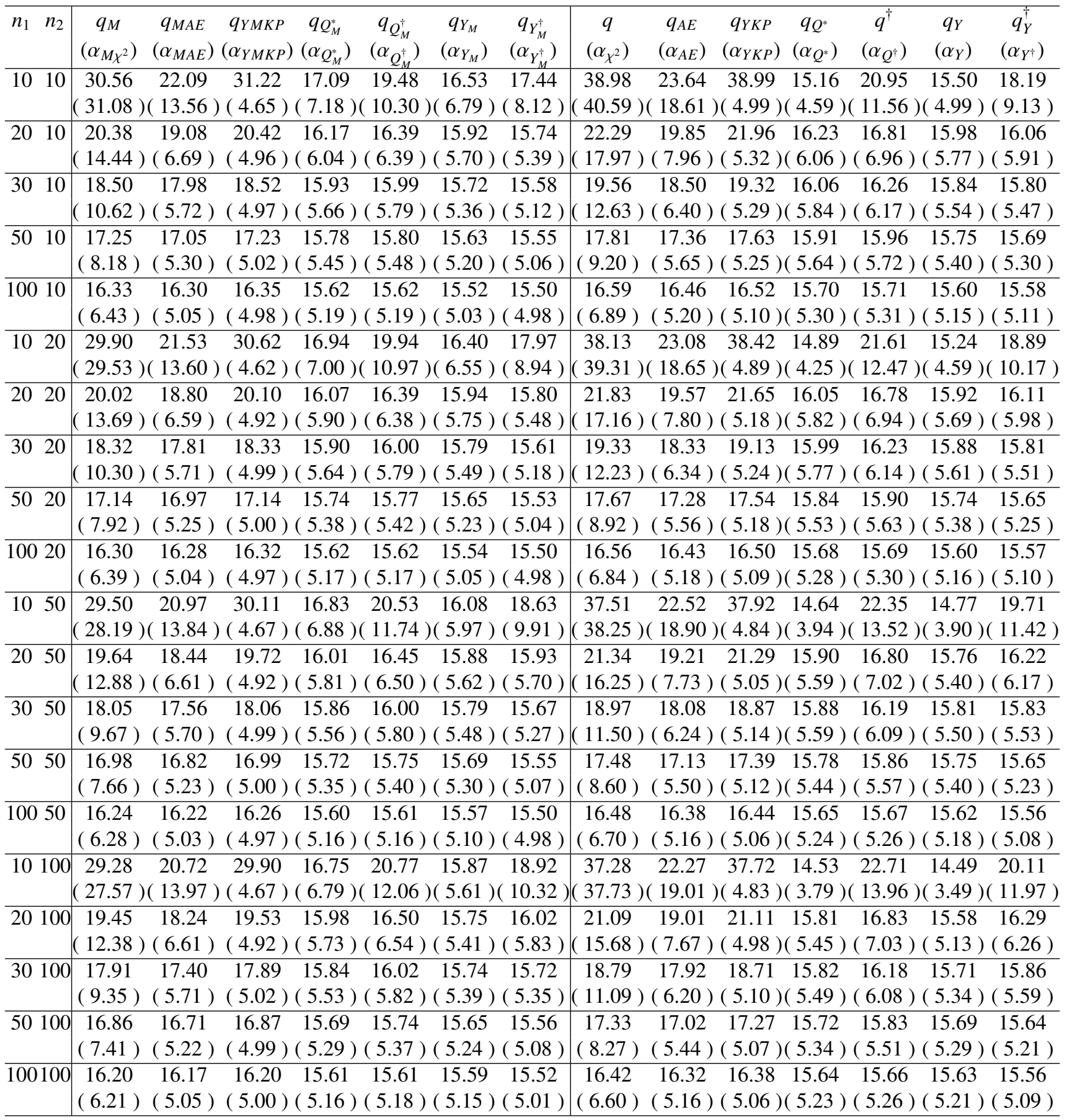

Note: $\chi_{8}^{2}(0.05)=15.51$ 
Table 9. The upper percentiles of test statistics and empirical Type I errors for the one sample case, $\left(n_{1}, n_{2}\right)=(30,30), p_{2}$ : fix

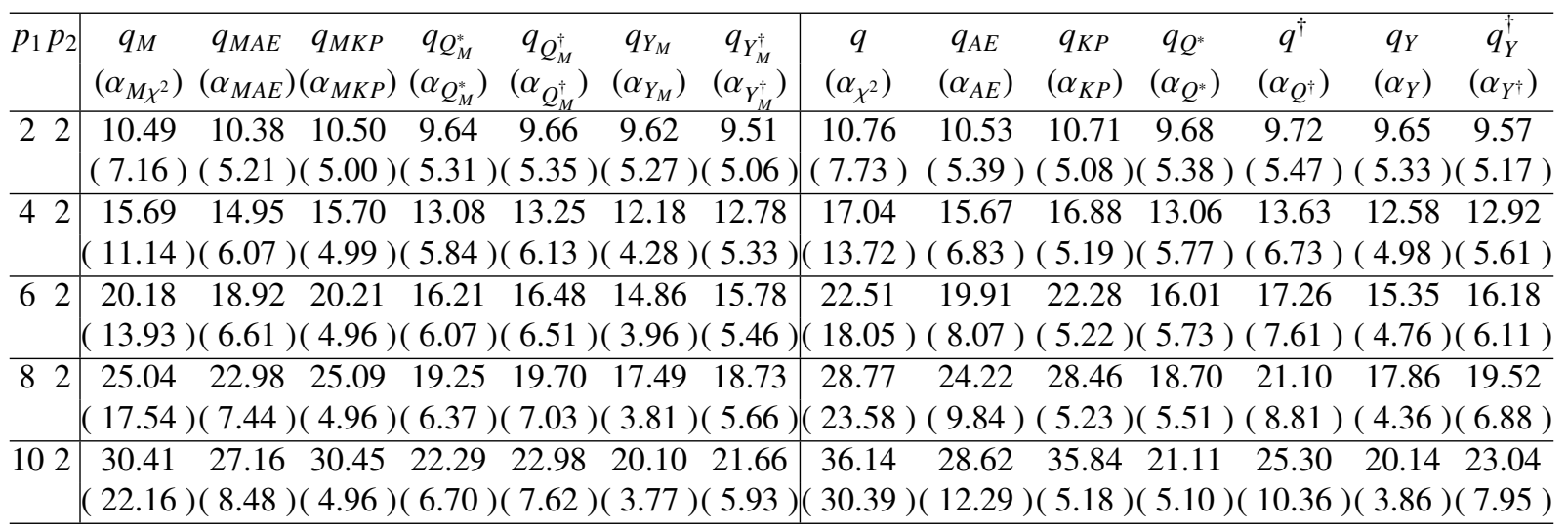

Note: $\chi_{4}^{2}(0.05)=9.49, \chi_{6}^{2}(0.05)=12.59, \chi_{8}^{2}(0.05)=15.51, \chi_{10}^{2}(0.05)=18.31, \chi_{12}^{2}(0.05)=18.31$

Table 10. The upper percentiles of test statistics and empirical Type I errors for the two sample case, $\left(n_{1}, n_{2}\right)=$ $(30,30), p_{2}:$ fix

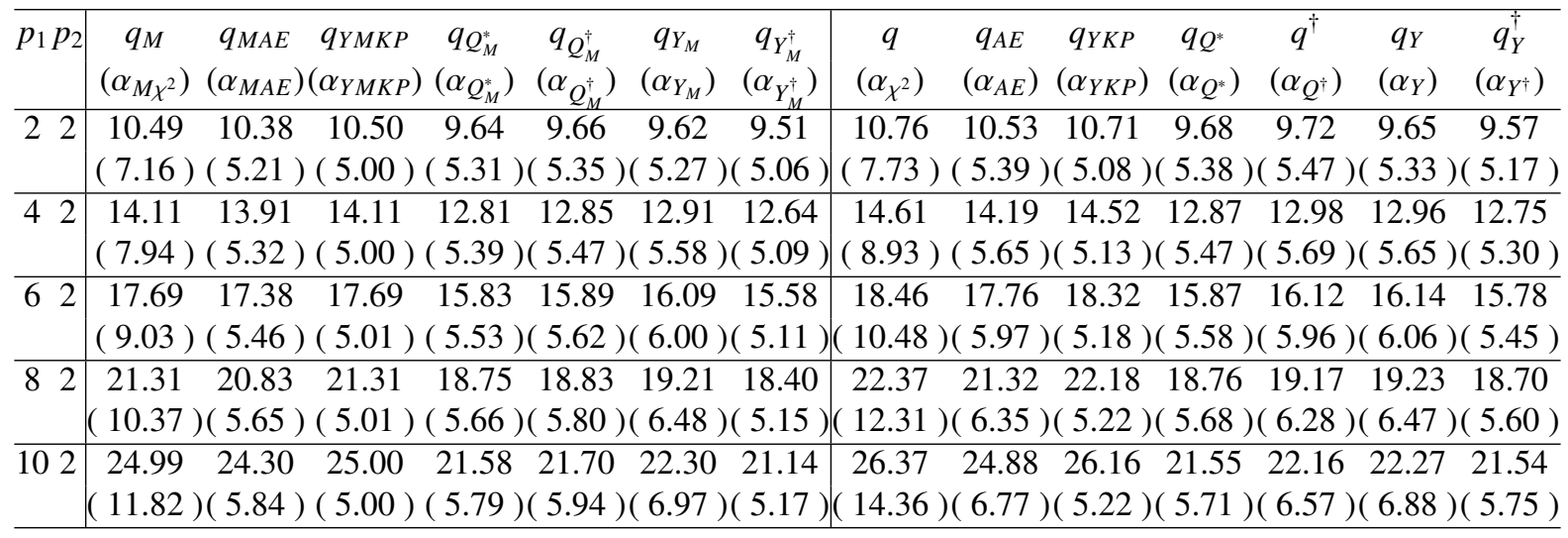

Note: $\chi_{4}^{2}(0.05)=9.49, \chi_{6}^{2}(0.05)=12.59, \chi_{8}^{2}(0.05)=15.51, \chi_{10}^{2}(0.05)=18.31, \chi_{12}^{2}(0.05)=18.31$

\section{Acknowledgements}

The second and third authors' research is partly supported by a Grant-in-Aid for Young Scientists (JSPS KAKENHI Grant Number JP19K20225) and a Grant-in-Aid for Scientific Research (C) (JSPS KAKENHI Grant Number JP17K00058), respectively.

\section{References}

Anderson, T. W. (2003). An Introduction to Multivariate Analysis. (3rd ed.). John Wiley \& Sons, Inc., New York.

Chang, W.-Y., \& Richards, D. St. P. (2009). Finite-sample inference with monotone incomplete multivariate normal data, I. Journal of Multivariate Analysis, 100, 1883-1899. https://doi.org/10.1016/j.jmva.2009.05.003

Fujikoshi, Y. (2000). Transformations with improved chi-squared approximations. Journal of Multivariate Analysis, 72 , 249-263. https://doi.org/10.1006/jmva.1999.1854

Jinadasa, K. G., \& Tracy, D. S. (1992). Maximum likelihood estimation for multivariate normal distribution with monotone sample. Communications in Statistics - Theory and Methods, 21, 41-50. https://doi.org/10.1080/03610929208830763

Krishnamoorthy, K., \& Pannala, M. K. (1998). Some simple test procedures for normal mean vector with incomplete data. Annals of the Institute of Statistical Mathematics, 50, 531-542. https://doi.org/10.1023/A:1003581513299

Krishnamoorthy, K., \& Pannala, M. K. (1999). Confidence estimation of a normal mean vector with incomplete data. The Canadian Journal of Statistics, 27, 395-407. https://doi.org/10.2307/3315648 
Morrison, D. F. (2005). Multivariate Statistical Methods. (4th ed.). Duxbury.

Seko, N., Kawasaki, T., \& Seo, T. (2011). Testing equality of two mean vectors with two-step monotone missing data. American Journal of Mathematical and Management Sciences, 31, 117-135. https://doi.org/10.1080/01966324.2011.10737802

Seko, N., Yamazaki, A., \& Seo, T. (2012). Tests for mean vector with two-step monotone missing data. SUT Journal of Mathematics, 48, 13-36. https://doi.org/10.20604/00000956

Yagi, A., \& Seo, T. (2017). Test for normal mean vectors with monotone incomplete data. American Journal of Mathematical and Management Sciences, 36, 1-20. http://dx.doi.org/10.1080/01966324.2016.1236712

Yagi, A., Seo, T., \& Hanusz. (2018). Testing equality of two mean vectors with monotone missing data. Technical Report No.18-07, Statistical Research Group, Hiroshima University, Hiroshima, Japan.

Yagi, A., Seo, T., \& Hanusz. (2019). Improved simplified $T^{2}$ test statistics for mean vector with monotone missing Data. Communications in Statistics-Simulation and Computation, 48(6), 1606-1622. https://doi.org/10.1080/03610918.2017.1419261

Yu, J., Krishnamoorthy, K., \& Pannala, M. K. (2006). Two-sample inference for normal mean vectors based on monotone missing data. Journal of Multivariate Analysis, 97, 2162-2176. https://doi.org/10.1016/j.jmva.2006.07.002

\section{Copyrights}

Copyright for this article is retained by the author(s), with first publication rights granted to the journal.

This is an open-access article distributed under the terms and conditions of the Creative Commons Attribution license (http://creativecommons.org/licenses/by/4.0/). 